Pacific Journal of Mathematics

ON SOME CLASSES OF NEARLY OPEN SETS 


\section{ON SOME CLASSES OF NEARLY OPEN SETS}

\section{OLAV NJÅSTAD}

The open sets in a topological space are those sets $A$ for which $A^{0} \supset A$. Sets for which $A^{0-0} \supset A$-" $\alpha$-sets"- or $A^{0-} \supset A$ -" $\beta$-sets"- may naturally be considered as more or less "nearly open". In this paper the structure of these sets and classes of sets are investigated, and some applications are given.

Topologies determining the same class of $\alpha$-sets also determine the same class of $\beta$-sets, and vice versa. The class of $\beta$-sets forms a topology if and only if the original topology is extremally disconnected. The class of $\alpha$-sets always forms a topology, and topologies generated in this way-" $\alpha$-topolgies"are exactly those where all nowhere dense sets are closed.

The class of all topologies which determine the same $\alpha$-sets is convex in the ordering by inclusion, the $\alpha$-topology being its finest member. Most topologies ordinary met with are the coarsest members of their corresponding classes; in particular this is the case for all regular topologies.

All topologies determining the same $\alpha$-sets also determine the same continuous mappings into arbitrary regular spaces.

Since the paper was submitted, it has come to our attention that Freud, in [5], has investigated problems which have a certain connection with parts of those here treated.

1. Let $\mathscr{T}$ be a topology (identified with its class of open sets) on a set $E$, and let ${ }^{0}$ and - denote interior and closure with respect to $\mathscr{T}$. We shall call a set $A$ with the property $A^{0-0} \supset A$ an $\alpha$-set (with respect to $\mathscr{T}$ ), and we shall denote the class of all such sets $\mathscr{T}^{a}$. A set $B$ with the property $B^{0-} \supset B$ (or equivalently $B^{0-}=B^{-}$) shall be called a $\beta$-set (with respect to $\mathscr{T}$ ), and the class of all such sets denoted $\mathscr{T}^{\beta}$. A class consisting of exactly all the $\alpha$-sets (resp. $\beta$-sets) of some topology shall be called an $\alpha$-structure (resp. $\beta$ structure). Evidently $\mathscr{T} \subset \mathscr{T}^{\alpha} \subset \mathscr{T}^{\beta}$. We notice that every nonempty $\beta$-set has a nonempty interior. If all sets of the family $\left\{B_{i}\right\}_{i \in I}$ are $\beta$-sets, then

$$
\bigcup_{i \in I} B_{i} \subset \bigcup_{i \in I} B_{i}^{0-} \subset\left(\bigcup_{i \in I} B_{i}^{0}\right)^{-} \subset\left(\bigcup_{i \in I} B_{i}\right)^{0-},
$$

that is: A $\beta$-structure is closed with respect to arbitrary unions.

We shall now characterize $\mathscr{T}^{\alpha}$ in terms of $\mathscr{T}^{\beta}$ : 
Proposition 1. Let $\mathscr{T}$ be a topology on $E$. $\mathscr{T}^{a}$ consists of exactly those sets $A$ for which $A \cap B \in \mathscr{T}^{\beta}$ for all $B \in \mathscr{T}^{\beta}$.

Proof. Let $A \in \mathscr{T}^{\alpha}, B \in \mathscr{T}^{\beta}, x \in A \cap B$ and let $U$ be an open neighbourhood of $x$. Clearly $U \cap A^{0-0}$ too is an open neighbourhood of $x$, so $V=\left(U \cap A^{0-0}\right) \cap B^{0}$ is nonempty. Since $V \subset A^{0-}$ this implies

$$
U \cap\left(A^{0} \cap B^{0}\right)=V \cap A^{0} \neq \phi .
$$

It follows that

$$
A \cap B \subset\left(A^{0} \cap B^{0}\right)^{-}=(A \cap B)^{0-},
$$

i.e. $A \cap B \in \mathscr{T}^{\beta}$.-Conversely, let $A \cap B \in \mathscr{T}^{\beta}$ for all $B \in \mathscr{T}^{\beta}$. Then in particular $A \in \mathscr{T}^{\beta}$. Assume $x \in A \cap \mathrm{C}\left(A^{0-0}\right)$ (C denoting complement). Then $x \in B^{-}$, where $B=\mathrm{C}\left(A^{0-}\right)$. Clearly $\{x\} \cup B \in \mathscr{T}^{\beta}$, and consequently

$$
A \cap(\{x\} \cup B) \in \mathscr{T}^{\beta} \text {. }
$$

But

$$
A \cap(\{x\} \cup B)=\{x\},
$$

hence $\{x\}$ is open. As $x \in A^{0-}$, this implies $x \in A^{0-0}$, contrary to assumption. Thus $x \in A$ implies $x \in A^{0=0}$, and $A \in \mathscr{T}^{\alpha}$. This completes the proof.

-Thus we have found that $\mathscr{T}^{\alpha}$ is completely determined by $\mathscr{T}^{-\beta}$, i.e.: all topologies with the same $\beta$-structue also determine the same $\alpha$-structure, explicitly given by Prop. 1 . We shall see that conversely all topologies with the same $\alpha$-structure determine the same $\beta$-structure, so that $\mathscr{T}^{\beta}$ is completely determined by $\mathscr{T}^{\alpha}$.

Proposition 2. Every $\alpha$-structure is a topology.

Proof. $\mathscr{T}^{\beta}$ contains the empty set and is closed with respect to arbitrary unions. A standard result gives that the class of those sets $A$ for which $A \cap B \in \mathscr{T}^{\beta}$ for all $B \in \mathscr{T}^{\beta}$ constitutes a topology, hence the proposition.

-Henceforth we shall also use the term $\alpha$-topology for $\alpha$-structure. Two topologies determining the same $\alpha$-structure shall be called $\alpha$-equivalent, and the equivalence classes shall be called $\alpha$-classes.

We may now characterize $\mathscr{T}^{\beta}$ in terms of $\mathscr{T}^{\alpha}$ in the following way:

Proposition 3. Let $\mathscr{T}$ be a topology on $E$. Then $\mathscr{T}^{\beta}=\mathscr{T}^{\alpha \beta}$, and hence $\alpha$-equivalent topologies determine the same $\beta$-structure. 
Proof. Let cl. and int. denote closure and interior with respect to $\mathscr{T}^{\alpha}$. If $x \in B \in \mathscr{T}^{\beta}$ and $x \in A \in \mathscr{T}^{\alpha}$, then $A^{0-0} \cap B^{0} \neq \phi$ since $A^{0-0}$ is a neighbourhood of $x$. So certainly $B^{0}$ meets $A^{0-}$ and therefore (being open) meets $A^{0}$, proving $A \cap B^{0} \neq \phi$, and $a$ fortiori $A \cap$ int. $B \neq \phi$. This means

$$
\text { cl. int. } B \supset B \text {, }
$$

i.e.: $B \in \mathscr{T}^{\alpha \beta}$. $\quad$ On the other hand let $A \in \mathscr{T}^{\alpha \beta}, x \in A$ and $x \in V \in \mathscr{T}$. As $V \in \mathscr{T}^{\alpha}$ and $x \in \operatorname{cl}$. int. $A$, we have

$$
V \cap \operatorname{int} . A \neq \phi,
$$

and there exists a nonempty set $W \in \mathscr{T}$ such that $W \subset V \cap$ int. $A \subset A$. In other words:

$$
V \cap A^{0} \neq \phi,
$$

and $x \in A^{0-}$. Thus we have verified $\mathscr{T}^{\alpha \beta} \subset \mathscr{T}^{\alpha}$, and the proof is complete. -Combining Prop. 1 and Prop. 3 we get $\mathscr{T}^{\alpha \alpha}=\mathscr{T}^{\alpha}$, or

COROLlary 1. A topology $\mathscr{T}$ is an $\alpha$-topology if and only if $\mathscr{T}=\mathscr{T}^{\alpha}$. Thus an $\alpha$-topology belongs to the $\alpha$-class of all its determining topologies, and is the finest topology of this class.

Evidently $\mathscr{T}^{\beta}$ is a topology if and only if $\mathscr{T}^{\alpha}=\mathscr{T}^{\beta}$. In this case $\mathscr{T}^{\beta \beta}=\mathscr{T}^{\alpha \beta}=\mathscr{T}^{\beta}$, or

CoRollary 2. If a $\beta$-structure $\mathscr{B}$ is a topology, then $\mathscr{B}=$ $\mathscr{B}^{\alpha}=\mathscr{B}^{\beta}$.

Before proceeding with our discussion we consider the following

ExAMPLE. Let $\mathscr{R}$ be the ordinary topology on the set of real numbers $R$. Clearly the complement of a nowhere dense, not closed set (such sets certainly exist) is a nonopen $\alpha$-set. So $\mathscr{R} \neq \mathscr{R}^{\alpha}$. Among the $\beta$-sets are all intervals, so $\mathscr{R}^{\alpha} \neq \mathscr{R}^{\beta}$, and $\mathscr{R}^{\beta}$ is not a topology.

2. We proceed to give some results on the structure of $\alpha$-topologies.

Proposition 4. The $\alpha$-sets with respect to a given topology are exactly those sets which may be written as a difference between an open set and a nowhere dense set.

Proof. If $A \in \mathscr{T}^{\alpha}$ we have 


$$
A=A^{0-0}-\left(A^{0-0}-A\right),
$$

where $A^{0-0}-A$ clearly is nowhere dense. Conversely, if $A=B-N$, $B \in \mathscr{T}, N$ nowhere dense, we easily see that $A^{0-} \supset B$ and consequently

$$
A^{0-0} \supset B \supset A \text {. }
$$

So the proof is complete.

COROLlaRY. A topology is an $\alpha$-topology if and only if all nowhere dense sets are closed.

For an $\alpha$-topology may clearly be characterized as a topology where the difference between an open set and a nowhere dense set is again an open set, and this evidently is equivalent to the condition stated.

Proposition 5. Topologies which are $\alpha$-equivalent determine the same class of nowhere dense sets.

Proof. Let $N$ be a nowhere dense set with respect to $\mathscr{T}^{a}$. For every nonempty $A \in \mathscr{T}$ there is a nonempty $B \in \mathscr{T}^{a}$ such that $B \subset A$ and $B \cap N=\phi$. As $B^{0} \neq \phi$, it follows that $N$ is not dense in $A$ with respect to $\mathscr{T}$, and consequently that $N$ is nowhere dense with respect to $\mathscr{T}$.

Conversely, let $N$ be nowhere dense with respect to $\mathscr{T} \cdot N^{-}$ contains no nonempty set from $\mathscr{T}$. As cl. $N \subset N^{-}$(cl. denotes closure with respect to $\mathscr{T}^{\alpha}$ ), cl. $N$ contains no nonempty set from $\mathscr{T}^{a}$. So $N$ is nowhere dense with respect to $\mathscr{T}^{\alpha}$, which completes the proof.

The converse of Prop. 5 is naturally not true, since both the coarsest and the finest topology on a set are $\alpha$-topologies, and neither of them possesses any nonempty nowhere dense sets.

We observe that every $\beta$-set- and $a$ fortior $i$ every $\alpha$-set- is the union of an open set and a nowhere dense set. This together with the corollary to Prop. 4 implies

COROLLARY 1. If the topology $\mathscr{T}$ has the property that all open sets are $\mathscr{F}_{\sigma}$-sets, then the topology $\mathscr{T}^{a}$ has the same property.

A topology where no nonempty open set is of the first category is called a Baire-topology (cf. [4, p. 109]). From what is said above immediately follows

CoRollary 2. A topology $\mathscr{T}$ is a Baire-topology if and only only if $\mathscr{T}^{a}$ is a Baire-topology. 
We shall comment on these corollaries later on.

A set $A$ is called minimally bounded with respect to the topology $\mathscr{T}$ if $A^{0-} \supset A, A^{-0} \subset A$ (cf. [1, p. 101]). (In the case of open sets, minimal boundedness coincides with regularity in the sense of $[2, \mathrm{p}$. 176]). Clearly this means $A \in \mathscr{T}^{\beta}, \mathrm{C} A \in \mathscr{T}^{\beta}$.

Proposition 6. Topologies which are $\alpha$-equivalent determine the same class of minimally bounded sets, and the same class of minimally bounded open sets.

Proof. The first assertion is obvious. Let $A \in \mathscr{T}^{\alpha}, \mathrm{C} A \in \mathscr{T}^{\beta}$. Then we have

$$
A^{0-0} \supset A, \quad(\mathrm{C} A)^{0-} \supset \mathrm{C} A
$$

It follows that

$$
A^{0-0}=A^{-0}=A \text {. }
$$

So $A \in \mathscr{T}$, and the proposition follows.

We recall that a topology is called extremally disconnected if the closure of every open set is open.

Proposition 7. If the $\beta$-structure $\mathscr{B}$ is a topology, all topologies $\mathscr{T}$ for which $\mathscr{T}^{\beta}=\mathscr{B}$ are extremally disconnected. If $\mathscr{B}$ is not a topology, no $\mathscr{T}$ for which $\mathscr{T}^{\beta}=\mathscr{B}$ is extremally disconnected. In particular: Either all or none of the topologies of an $\alpha$-class are extremally disconnected.

Proof. Let $\mathscr{T}^{\beta}=\mathscr{B}$, and suppose there is an $A \in \mathscr{T}^{-}$such that $A^{-} \notin \mathscr{T}$. Let $x \in A^{-}-A^{-0}$. With

$$
B=\{x\} \cup A^{-0}, C=\mathrm{C}\left(A^{-0}\right)
$$

we have

$$
B^{0-} \supset A^{-0-}=A^{-} \supset\{x\}, C^{0-}=\mathrm{C}\left(A^{-0}\right)=C \supset\{x\} .
$$

Hence both $B$ and $C$ are in $\mathscr{T}^{\beta}$. The intersection $B \cap C=\{x\}$ is not open since $x \in A^{-}-A^{-0}$, hence not a $\beta$-set. So $\mathscr{B}=\mathscr{T}^{\beta}$ is not a topology.

Now suppose $\mathscr{B}$ is not a topology, and $\mathscr{T}^{\beta}=\mathscr{B}$. There is a $B \in \mathscr{T}^{\beta}$ such that $B \notin \mathscr{T}^{\alpha}$. Asssume $B^{0-} \in \mathscr{T}$. Then

$$
B^{0-0}=B^{0-} \supset B,
$$


i.e. $B \in \mathscr{T}^{a}$, contrary to assumption. Thus we have produced an open set whose closure is not open, which completes the proof.

COROLlary. A topology $\mathscr{T}$ is extremally disconnected if and only if $\mathscr{T}^{\beta}$ is a topology.

Proof evident.

We next give a result on the continuous mappings of the topological space $(E, \mathscr{T})$ into a topological space $(F, \mathscr{U})$.

Proposition 8. All topologies of a given $\alpha$-class on $E$ determine the same class of continuous mappings into an arbitrary regular topological space $(F, \mathscr{U})$.

Proof. Let $\mathscr{T}$ be a topology on $E$ and assume $f$ continuous from $\left(E, \mathscr{T}^{\alpha}\right)$ into the regular space $(F, \mathscr{U})$. Let $x \in E$, and let $U$ be a closed neighbourhood of $f(x)$. There is a $V \in \mathscr{T}^{a}$ such that $x \in V$ and $f(V) \subset U$. We may write $V=A-N$, where $A \in \mathscr{T}, N$ is nowhere dense (Prop. 4), $N \subset A$. Let $y \in N$, and suppose $f(y) \notin U$. Then there exists a $W \in \mathscr{T}^{\alpha}$ such that $y \in W \subset A$ and $f(W) \subset \subset U$. But as $N$ is nowhere dense we have $W \not \subset N$. That is: $\mathrm{W} \cap V \neq \phi$, contrary to assumption. Thus $f(A) \subset U$, hence $f$ is continuous with respect to $\mathscr{T}$ and $\mathscr{U}$ and the proof is complete.

In this connection we mention that a mapping of the space $(E, \mathscr{T})$ into the space $(F, \mathscr{U})$ is called quasicontinuous if for every $x \in E$, every neighbourhood $V$ of $x$ and $U$ of $f(x)$ there exists a nonempty $W \in \mathscr{T}$ such that $W \subset V$ and $f(W) \subset U$ (cf. [6, p. 184]). It is easily seen that this is equivalent to the condition: For every $U \in \mathscr{U}$, $f^{-1}(U) \in \mathscr{T}^{-\beta}$. Hence we immediately get

Proposition 9. All topologies of a given $\alpha$-class on $E$ determine the same class of quasicontinuous mappings into an arbitrary topological space $(F, \mathscr{U})$.

3. We shall now deduce some properties of the order structure of the $\alpha$-classes. We first recall that every class contains a finest element, namely its associated $\alpha$-topology. Next we prove the following

Proposition 10. Every $\alpha$-class is convex in the set of topologies on $E$ ordered by inclusion. That is: $\mathscr{T} \subset \mathscr{U} \subset \mathscr{T}^{\alpha}$ implies $\mathscr{T}^{a}=\mathscr{U}^{a}$.

Proof. We assume $\mathscr{T} \subset \mathscr{U} \subset \mathscr{T}^{\alpha}$, and denote interior and closure in $\mathscr{T}$ (resp. $\mathscr{U})$ by ${ }^{0},-$ (resp. int., cl.). Using to denote closure 
in $\mathscr{T}^{a}$, we have (for all $A$ )

$$
\text { cl. int. } A \supset(\text { int. } A)^{\sim} \supset A^{0 \sim} \text {. }
$$

Now $A^{0 \sim}$ is the closure of an open set in $\mathscr{T}^{\alpha}$, hence minimally bounded in $\mathscr{T}^{a}$ and so also in $\mathscr{T}$. Hence

$$
A^{0 \sim} \supset A^{0 \sim-0} \supset A^{0-0},
$$

so that

$$
\text { cl. int. } A \supset A^{0-0} \text {. }
$$

It follows that

$$
\text { int. cl. int. } A \supset A^{0-0} \text {. }
$$

Hence, if $A \in \mathscr{T}^{\alpha}$, we have $a$ fortiori that

$$
\text { int. cl. int. } A \supset A \text {. }
$$

Thus

$$
\mathscr{U} \subset \mathscr{T}^{a} \subset \mathscr{U}^{a} \text {. }
$$

Applying this result to the inclusion $\mathscr{U} \subset \mathscr{T}^{\alpha} \subset \mathscr{U}$ we get

$$
\mathscr{T}^{\alpha} \subset \mathscr{U}^{\alpha} \subset \mathscr{T}^{\alpha \alpha}=\mathscr{T}^{\alpha} .
$$

So the equality $\mathscr{T}^{\alpha}=\mathscr{Q}^{\alpha}$ is proved.

We shall now investigate in some detail the problem of a coarsest topology in the $\alpha$-class, and in this connection give conditions for an $\alpha$-topology to be the only topology of its class. To this end we make the following definition: We shall say that the topology $\mathscr{T}$ is quasiregular at a point $x$ if the point has a fundamental system of minimally bounded open neighbourhoods, and call the topology quasi-regular if it is quasi-regular at all points. It is easily seen that an equivalent condition for quasi-regularity at the point $x$ is that it has a fundamental system of interiors of closed neighbourhoods. Clearly every topology satisfying the separation axiom $0_{I I I}$ of $[3, \mathrm{p}$. 54] is quasi-regular, in particular every regular topology. With this terminology we may characterize those topologies which are the coarsest members of their classes in the following way:

Proposition 11. A topology $\mathscr{T}$ is the coarsest topology of its $\alpha$-class if and only if it is quasi-regular at all points which are closed with respect to $\mathscr{T}^{\alpha}$. 
Proof. All topologies $\mathscr{U}$ for which $\mathscr{U}^{a}=\mathscr{T}^{a}$ determine the same class of minimally bounded open sets, and therefore the neighbourhood filter with respect to $\mathscr{T}$ is coarser than that with respect to $\mathscr{U}$ at points where $\mathscr{T}$ is quasi-regular. Now assume that $\mathscr{T}$ is quasiregular at all points which are closed with respect to $\mathscr{T}^{-\alpha}$, and let $x$ be a point where $\mathscr{T}$ is not quasi-regular. Then $\{x\}$ is not nowhere dense, i.e. int.cl. $\{x\} \neq \phi$ (int. and cl. denote interior and closure with respect to $\mathscr{T}^{\alpha}$ ). Every nonempty $\alpha$-set contained in int. cl. $\{x\}$ clearly contains $x$. Now every such set contains a nonempty set from $\mathscr{U}$, which of course also contains $x$. It follows that the neighbourhood filter with respect to $\mathscr{U}$ constitutes a fundamental system of neighbourhoods with respect to $\mathscr{T}^{a}$. This means that all topologies of the $\alpha$-class possess the same neighbourhood filter at $x$. Thus the sufficiency of the condition is demonstrated.

Now let $\{x\}$ be closed with respect to $\mathscr{T}^{\alpha}$, and suppose that $\mathscr{T}$ (and hence $\mathscr{T}^{\alpha}$ ) is not quasi-regular at $x$. Let $\mathscr{Y}(x)$ denote the filter generated by all minimally bounded open neighbourhoods of $x$, and $\mathscr{Y}^{-}(y)$ the neighbourhood filter of $y$ with respect to $\mathscr{T}^{a}$ for $y \neq x$. It is easily verified that $\mathscr{Y}(z)$ satisfies the requirements $V_{I}-V_{I V}$ of [3, p. 11-12] for all $z \in E$. Thus the filters $\mathscr{V}(z)$ determine a topology $\mathscr{U}$ on $E$, which is not finer than $\mathscr{T}$, since the neighbourhood filter of $x$ with respect to $\mathscr{U}$ is strictly coarser than that with respect to $\mathscr{T}$. Obviously $\mathscr{U} \subset \mathscr{T}^{\alpha}$.

Let - and ${ }^{0}$ denote closure and interior with respect to $\mathscr{C}$. Closures with respect to $\mathscr{T}^{a}$ and with respect to $\mathscr{C}$ of sets containing $x$ clearly are equal. Now assume $A \in \mathscr{T}^{a}$. Our object is to prove $A \in \mathscr{C}^{a}$. Suppose this is false; then $A \notin \mathscr{Q}$, so $A$ is an open neighbourhood of $x$ with respect to $\mathscr{T}^{a}$. Clearly $A^{0}=A-\{x\}$. Now from $x \in A^{0-}$ would follow

$$
A^{0-}=\text { cl. } A \supset \text { int. cl. } A \supset A \supset\{x\} \text {. }
$$

Since int. cl. $A$ is minimally bounded it belongs to $\mathscr{C}$, and we would have

$$
A^{0-0} \supset \text { int. cl. } A \supset A
$$

which means $A \in \mathscr{Q}^{a}$. Thus we conclude that $x \notin A^{0-}$. Then there is a $B \in \mathscr{C}$ containing $x$ such that $B \cap(A-\{x\})=\phi$. Since both $A$ and $B$ are contained in $\mathscr{T}^{\alpha}, A \cap B \in \mathscr{T}^{\alpha}$. But $A \cap B=\{x\}$, and $\{x\}$ is closed with respect to $\mathscr{T}^{\alpha}$. This would mean that $\mathscr{T}^{\infty}$ is quasi-regular at $x$, contrary to assumption. So we may conclude that $A \in \mathscr{U}^{a}$. Thus we have shown that $\mathscr{T}^{\alpha} \subset \mathscr{U}^{\alpha}$. From $\mathscr{U} \subset \mathscr{T}^{\alpha} \subset \mathscr{U}^{\alpha}$ we conclude $\mathscr{C}^{\alpha}=\mathscr{T}^{\alpha \alpha}=\mathscr{T}^{\alpha}$ (Prop. 10). Thus if $\mathscr{T}$ is not quasi-regular at all 
points which are closed with respect to $\mathscr{T}^{\alpha}$, it is not the coarsest topology of its $\alpha$-class. This completes the proof.

COROLlary 1. A quasi-regular topology $\mathscr{T}$ is the coarsest topology of its $\alpha$-class.

Obvious.

CoRollary 2. If the topology $\mathscr{T}$ is not an $\alpha$-topology, then its $\alpha$-topology is not quasi-regular.

\section{Obvious.}

We see that from every topology which is itself not an $\alpha$-topology is deduced an $\alpha$-topology which in a certain sense is rather pathological. This may be of some interest, since $\mathscr{T}^{\alpha}$ also inherits some "nice" properties from $\mathscr{T}$. Thus consider the most usual Baire-spaces: those which are locally compact or deduced from a complete metric structure. Many of these are not $\alpha$-topologies; in these cases the construction of the associated $\alpha$-topology gives Baire-spaces which are not even uniformizable (cor. 2 to Prop. 5). And to every perfectly normal topology is associated an $(\alpha-)$ topology which may be far from being perfectly normal, but which nevertheless has the property that every open set is an $\mathscr{F}_{\sigma}$-set (Cor. 1 to Prop. 5). According to Prop. 8 these two topologies determine the same realvalued continuous functions, only the open sets of the first occurring as inverse images of open sets in $R$.

An $\alpha$-class does not always possess a coarsest topology, as is shown by the following

ExAMPLE. Let $E$ be an infinite set, and let $\mathscr{T}$ consist of the empty set and all complements of finite sets. This topology clearly is an $\alpha$-topology (and even a $\beta$-structure), not quasi-regular at any point. Here it is easily seen that the only topology which is coarser than all topologies of the $\alpha$-class determined by $\mathscr{T}$ is the coarsest topology on $E$. This topology itself does not belong to the class.

Proposition 12. An $\alpha$-topology $\mathscr{T}$ is the only topology of its $\alpha$-class if and only if it is quasi-regular at all closed points.

Proof evident.

As examples of $\alpha$-topologies which are the only topologies of their $\alpha$-classes we may mention the discrete topology and the coarsest 
topology on $E$ (both of which are quasi-regular). We have seen that the $\alpha$-topology deduced from the ordinary topology $\mathscr{R}$ on $R$ is distinct from $\mathscr{R}$, so this topology is not quasi-regular.

\section{REFERENCES}

1. G. Aumann, Reelle Funktionen, Berlin-Göttingen-Heidelberg, 1954.

2. G. Birkhoff, Lattice Theory, 2. ed., New York 1948.

3. N. Bourbaki, Topologie Générale, Ch. I-II, Act. Sci. et Ind. 858-1142, Paris 1951.

4. N. Bourbaki, Topologie Générale, Ch. IX, Acta. Sci. et Ind. 1045, Paris 1958.

5. G. Freud, Ein Beitrag zu dem Satze von Cantor und Bendixson, Acta. Math. Acad. Sci. Hung. 9 (1958), 333-336.

6. S. Kempisty, Sur les fonctions quasicontinues, Fund. Math. 19 (1932), 184-197. 


\section{PACIFIC JOURNAL OF MATHEMATICS}

\section{EDITORS}

\author{
H. Samelson \\ Stanford University \\ Stanford, California \\ R. M. Blumenthal \\ University of Washington \\ Seattle, Washington 98105
}

\author{
J. Dugundu \\ University of Southern California \\ Los Angeles, California 90007 \\ *Richard Arens \\ University of California \\ Los Angeles, California 90024
}

\section{ASSOCIATE EDITORS}
E. F. BeCKENBACH
B. H. NeUMANN
F. WOLF
K. YosidA

\section{SUPPORTING INSTITUTIONS}

\author{
UNIVERSITY OF BRITISH COLUMBIA \\ CALIFORNIA INSTITUTE OF TECHNOLOGY \\ UNIVERSITY OF CALIFORNIA \\ MONTANA STATE UNIVERSITY \\ UNIVERSITY OF NEVADA \\ NEW MEXICO STATE UNIVERSITY \\ OREGON STATE UNIVERSITY \\ UNIVERSITY OF OREGON \\ OSAKA UNIVERSITY \\ UNIVERSITY OF SOUTHERN CALIFORNIA
}

\author{
STANFORD UNIVERSITY \\ UNIVERSITY OF TOKYO \\ UNIVERSITY OF UTAH \\ WASHINGTON STATE UNIVERSITY \\ UNIVERSITY OF WASHINGTON \\ * * * * \\ AMERICAN MATHEMATICAL SOCIETY \\ CALIFORNIA RESEARCH CORPORATION \\ SPACE TECHNOLOGY LABORATORIES \\ NAVAL ORDNANCE TEST STATION
}

Mathematical papers intended for publication in the Pacific Journal of Mathematics should by typewritten (double spaced). The first paragraph or two must be capable of being used separately as a synopsis of the entire paper. It should not contain references to the bibliography. No separate author's resumé is required. Manuscripts may be sent to any one of the four editors. All other communications to the editors should be addressed to the managing editor, Richard Arens, at the University of California, Los Angeles, California 90024.

50 reprints per author of each article are furnished free of charge; additional copies may be obtained at cost in multiples of 50 .

The Pacific Journal of Mathematics is published quarterly, in March, June, September, and December. Effective with Volume 13 the price per volume (4 numbers) is $\$ 18.00$; single issues, $\$ 5.00$. Special price for current issues to individual faculty members of supporting institutions and to individual members of the American Mathematical Society: $\$ 8.00$ per volume; single issues $\$ 2.50$. Back numbers are available.

Subscriptions, orders for back numbers, and changes of address should be sent to Pacific Journal of Mathematics, 103 Highland Boulevard, Berkeley 8, California.

Printed at Kokusai Bunken Insatsusha (International Academic Printing Co., Ltd.), No. 6, 2-chome, Fujimi-cho, Chiyoda-ku, Tokyo, Japan.

PUBLISHED BY PACIFIC JOURNAL OF MATHEMATICS, A NON-PROFIT CORPORATION

The Supporting Institutions listed above contribute to the cost of publication of this Journal, but they are not owners or publishers and have no responsibility for its content or policies.

* Basil Gordon, Acting Managing Editor until February 1, 1966. 


\section{Pacific Journal of Mathematics}

\section{Vol. 15, No. $3 \quad$ November, 1965}

David R. Arterburn and Robert James Whitley, Projections in the space of

bounded linear operators .................................

Robert McCallum Blumenthal, Joram Lindenstrauss and Robert Ralph Phelps,

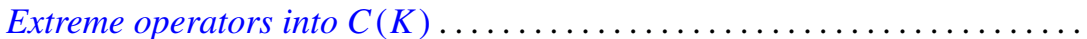

L. Carlitz, A note on multiple exponential sums ................... 757

Joseph A. Cima, A nonnormal Blaschke-quotient .................... 767

Paul Civin and Bertram Yood, Lie and Jordan structures in Banach algebras . . . 775

Luther Elic Claborn, Dedekind domains: Overrings and semi-prime

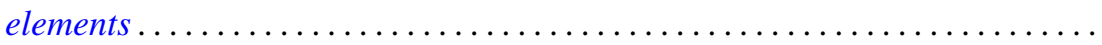

799

Luther Elic Claborn, Note generalizing a result of Samuel's .............. 805

George Bernard Dantzig, E. Eisenberg and Richard Warren Cottle, Symmetric

dual nonlinear programs ................................... 809

Philip J. Davis, Simple quadratures in the complex plane ............... 813

Edward Richard Fadell, On a coincidence theorem of F. B. Fuller ............ 825

Delbert Ray Fulkerson and Oliver Gross, Incidence matrices and interval

graphs ........................................ 835

Larry Charles Grove, Tensor products over $H^{*}$-algebras ..................

Deborah Tepper Haimo, $L^{2}$ expansions in terms of generalized heat polynomials

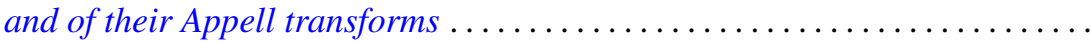

I. Martin (Irving) Isaacs and Donald Steven Passman, A chardcterization of groups in terms of the degrees of their characters ..........

Donald Gordon James, Integral invariants for vectors over local fields........ 905

Fred Krakowski, A remark on the lemma of Gauss ................... 917

Marvin David Marcus and H. Minc, A subdeterminant inequality ........... 921

Kevin Mor McCrimmon, Norms and noncommutative Jordan algebras ........ 925

Donald Earl Myers, Topologies for Laplace transform spaces ............... 957

Olav Njstad, On some classes of nearly open sets .................... 961

Milton Philip Olson, A characterization of conditional probability ........... 971

Barbara Osofsky, A counter-example to a lemma of Skornjakov .............. 985

Sidney Charles Port, Ratio limit theorems for Markov chains ............... 989

George A. Reid, A generalisation of $W^{*}$-algebras ...................... 1019

Robert Wells Ritchie, Classes of recursive functions based on Ackermann's

function ........................................... 1027

Thomas Lawrence Sherman, Properties of solutions of $n$th order linear

differential equations ........................................ 1045

Ernst Snapper, Inflation and deflation for all dimensions . .............. 1061

Kondagunta Sundaresan, On the strict and uniform convexity of certain Banach

spaces............................................. 1083

Frank J. Wagner, Maximal convex filters in a locally convex space .......... 1087

Joseph Albert Wolf, Translation-invariant function algebras on compact 